\title{
Enfoque social de la inclusión de arreglos silvopastoriles dentro de comunidades penitenciarias en Colombia
}

Social inclusion focus of silvopastoral arrangements within prison communities in Colombia

Martínez BJ ${ }^{1}$

${ }^{1}$ Esp. Escuela de Ciencias Agrícolas Pecuarias y Ambientales ECAPMA. UNAD javier.martinez@unad.edu.com

CONFERENCIA DEL I SEMINARIO INTERNACIONAL DE GANADERÍA AGROECOLÓGICA REALIZADO EN VILLAVICENCIO COLOMBIA, 11 y 12 de noviembre de 2010

\section{RESUMEN}

La Colonia Agrícola como establecimiento carcelario en el país, es quizás la referencia histórica de los procesos de encarcelamiento organizado, en la cual ha existido la intensión siempre de ser pionera en procesos productivos y de intervención terapéutica, desde su creación ha realizado esfuerzo por presentar propuestas de producción ganadera, tanto que este sistema ha surgido después de la tala y quema de los bosques, resultando en agroecosistemas con una escasa cobertura arbórea, con suelos desprotegidos y a menudo carentes de diversidad, al privilegiarse únicamente las pasturas. Esto ha permitido; que las áreas de pastoreo se vuelvan especialmente susceptibles a procesos erosivos, más aún cuando se encuentran en zonas de ladera. Al mismo tiempo; la producción ganadera en forma tradicional, implica generalmente una alta dependencia de insumos externos para rendir, incrementando los costos de producción y ocasionando ambientales. En este sentido, la búsqueda de sistemas de producción ganaderos más sustentables tanto ecológica como económicamente; además de ser socialmente aceptables y fácilmente adoptables, se hace una prioridad. Dentro de este contexto; los sistemas agroecológicos parecen ser una alternativa a corto, mediano y/o largo plazo capaz de cumplir con las demandas actuales de producción. En la actualidad se ha evaluado la inclusión social de arreglos silvopastoriles en la Colonia, como alternativa de tratamiento penitenciario, donde el interno realiza su plan de cambio, mediante la capacitación y la practica en ganadería agroecológica, experiencia recogida en el periodo de 2005 al 2010. 
Palabras clave: Cárceles, Colombia, rehabilitación.

\begin{abstract}
The Agricultural Colonia is a correctional facility in Colombia, it is perhaps the historical reference to organized processes of incarceration, where the intention has always been a pioneer in production processes and therapeutic intervention, since its inception has made effort to present proposals livestock production, while this system has emerged after cutting and burning of forests, resulting in agricultural ecosystems with little tree cover, soils unprotected and often lacking in diversity, unique privileges pastures. This has allowed, that the grazing areas become especially susceptible to erosion, especially when they are on slopes. At the same time, livestock production in traditional form, usually involves a high dependence on external inputs to yield, increasing production costs and causing environmental concerns. In this sense, the search for livestock production systems more sustainable both ecologically and economically as well as being socially acceptable and easily adoptable, it is a priority. Within this context, agro-ecological systems appear to be an alternative to short, medium and/or long term, being able to meet current demands of production. It is now assessed the social inclusion of silvopastoral arrangements in the colony, as an alternative treatment in prisons, where the inmate makes his bread exchange, through training and practice in livestock agro-ecological experience gained in the period from 2005 to 2010.
\end{abstract}

Keywords: Prisons, Colombia, rehabilitation.

\title{
INTRODUCCIÓN
}

La experiencia que se presenta en el documento, ha sido el producto de cinco años, de esfuerzos por buscar la comprensión de la problemática de Colombia, que termina en las cárceles, sin que nadie se interese por los 150.000 internos que purgan su condena, sin expectativa de vida, sin luz de esperanza, para que la sociedad los acepte nuevamente y por lo menos no volver pronto a las cárceles. El trabajo de campo se ha realizado, gracias al apoyo de algunos visionarios del sistema Penitenciario, como lo es el doctor Daniel Ortiz Mendoza, el Capitán Álvaro Peña y el doctor Dayro Cortes, quienes han 
comprendido que el desarrollo social y económico del país, no puede estar ajeno a la realidad de las cárceles, las cuales se convierten en un indicador de buena interpretación de la administración del recurso económico y de la comprensión del ser humano y su diferencia.

Lo interesante del trabajo realizado es la estructuración de un modelo de intervención terapéutica, basado en la actividad ocupacional, denominada Ganadería con bases Agroecológicas, donde el individuo, realiza su plan de tratamiento, para ser aceptado nuevamente por la sociedad, de esta manera las personas privadas de la libertad validan sus proyectos de vida, y se promociona la implementación de arreglos silvopastoriles en todo el país, donde ganamos todos, porque podemos contribuir con la seguridad alimentaria de la población. El modelo está diseñado con un tiempo no mayor a un año, situación que obliga a los profesionales a ser realmente eficientes. Al final del documento se cuentan las experiencias no tradicionales de la implementación del modelo de intervención basado en ganadería agroecológica.

\section{OBJETIVOS}

- Socializar experiencias en la implementación de un modelo de intervención terapéutica, basado en la actividad ocupacional, denominada ganadería con bases agroecológicas, en la Colonia Agrícola.

- Presentar la manera como se incluyeron arreglos silvopastoriles, en modelos de intervención terapéutica, para las personas privadas de la libertad en Colombia.

- Determinar los inconvenientes presentados en la experiencia de implementación, como modelo terapéutico.

\section{UBICACIÓN}

La experiencia plasmada en este documento, fue obtenida en la Colonia Agrícola de Acacias Meta, establecimiento carcelario, adscrito al Insttituto Nacional Penitenciario y Carcelario INPEC, la cual se encuentra ubicada en el departamento del Meta, en el municipio de Acacias, 3 kilómetros vía Acacias Villavicencio, en el periodo comprendido entre el año 2005 y el año 2010. 


\section{ENFOQUE TRADICIONAL DE LA GANADERÍA AGROECOLÓGICA}

El modelo convencional no utiliza adecuadamente el suelo como el factor más importante y fundamental del proceso productivo y lo lleva hacia una situación de hidroponía, externamente sostenida, a través de la aplicación de altas dosis de fertilizantes químicos de rápida solubilidad, con los problemas inherentes que esto trae para la biota y la naturaleza misma del suelo.

Así, este modelo ha llevado al productor o ganadero a una alta dependencia de insumos externos (herbicidas, insecticidas, vermífugos, baños, y otros), a la dilapidación de los recursos naturales, a la contaminación de las aguas, al envenenamiento de los alimentos, con sus repercusiones en la salud humana, al empobrecimiento del hombre del campo, al éxodo rural, al crecimiento de las ciudades y sus problemas de abastecimiento de agua, energía eléctrica, alimentos, transporte y seguridad. En el enfoque convencional, no se trabaja el sistema productivo como un todo, sino que se trabaja sólo sobre algunos de sus factores, como genética, fertilización, control de malezas, alimentación y otros aspectos. El modelo ha sobrepasado, tanto la capacidad fisiológica de los animales y plantas, como la capacidad agronómica de las fincas; por tanto, se ha dejado de producir con base a pasto y se ha convertido en un sistema reciclador de alimentos concentrados, con muy poca utilización del suelo, mediante la producción de forraje hidropónico en monocultivo.

El proceso de producción debe ser llevado a cabo respetando el bienestar y confort del animal, para que la explotación sea racional. Este bienestar está estrechamente relacionado con la calidad biológica de los productos obtenidos, con mayores y mejores niveles de producción, y con mayores beneficios económicos. Todo el proceso debe estar enmarcado en una atmósfera etológica, de respeto al bienestar del animal (Pinheiro, 2004). Por esto, los potreros deben contar con muy buena diversidad vegetal (ensalada en oferta), con el fin de brindarle al animal la posibilidad de balancear su dieta a través del año, con muy buena arborización en toda su extensión, brindándole también al animal condiciones adecuadas para realizar las operaciones propias del proceso de pastoreo, como la ingestión de forraje, la rumia, la digestión y sus funciones metabólicas en condiciones de temperatura confortable. 
En la agricultura agroecológica se maneja una visión más holística, más general, de todo el ecosistema. Se trabaja sobre las causas y no sobre los síntomas o efectos (Primavesi, 2002). En este enfoque se estudian los fundamentos y las bases de los diferentes procesos y fenómenos naturales, para, de esa manera, actuar en la práctica del proceso productivo, en el manejo y conservación del suelo y del agua, en el manejo de los pastos, de los animales, bajo la dirección inteligente del hombre. Se pone más énfasis en los aspectos ecológicos del suelo y en cómo desencadenar la biocenosis del mismo, el nuevo concepto de las arvenses o plantas indicadoras, la teoría de la trofobiosis, la transmutación de elementos a baja energía, la importancia crucial de reponerle al suelo la materia orgánica, el ciclo etileno, la acción solubilizadora del ácido carbónico y el favorecimiento de la biota del suelo (Pinheiro, 2004).

La esencia conceptual de este enfoque agroecológico de la ganadería está basada en la maximización de la captación de energía solar, que no tiene costo, es infinita, y utilizada en la fotosíntesis, no contamina el ambiente durante su transformación en energía biológica, esto es, en forma de carne, leche, lana, pieles, trabajo y demás utilidades limpias para la satisfacción de las necesidades de la humanidad. Hoy a nivel mundial, se busca desarrollar conductas agroecológicas y más sostenibles y sustentables. En la ganadería ecológica el sistema de manejo de los potreros, se basa en la intervención del hombre en los procesos de la vida de los pastos, de la vida de los animales, de la vida del ambiente, comenzando por la vida del suelo y el desarrollo de la biocenosis, que es el desarrollo dinámico de la vida del suelo.

En la ganadería ecológica los diversos nutrientes que requieren los pastos, se encuentran en la biomasa vegetal de la hojarasca diversa que cubre el suelo, proveniente de plantas de raíces profundas que reciclan estos nutrientes. La ganadería ecológica es un sistema dinámico, donde todos sus elementos están interrelacionados y en continuo cambio, de tal manera que cualquier acción sobre uno de sus componentes se refleja en los demás, cuyos efectos no siguen un patrón predeterminado (Pinheiro, 2004). En la ganadería ecológica no se utilizan elementos contaminantes de los productos obtenidos, ni del 
ambiente; la producción es completamente natural y no causa efectos dañinos en el ambiente.

\section{INCLUSIÓN DE ARREGLOS SILVOPASTORILES DENTRO DE COMUNIDADES PENITENCIARIAS EN COLOMBIA}

\section{Metodología}

La experiencia se realiza en tres fases, de la siguiente manera: La fase uno inicia en el año 2005, con la realización de un diagnóstico de la población reclusa a nivel nacional, donde se encontraron datos del crecimiento avanzado de la población privada de la libertar, y que además un porcentaje significativo tenía vocación productiva en el sector ganadero, como se aprecia en el Cuadro 1, donde se entregan datos por regionales del INPEC, del número de personas privadas de la libertad que se encuentran en las cárceles.

Cuadro 1. Cuadro estadístico de personas privadas de la libertad

\begin{tabular}{lcccc}
\hline \multicolumn{1}{c}{ REGIONAL } & CAPACIDAD & \multicolumn{3}{c}{ TOTAL POBLACIÓN } \\
\hline REGIONAL CENTRAL & 20.919 & 28.207 & 28.226 & 28.256 \\
REGIONAL OCCIDENTE & 13.059 & 13.323 & 14.938 & 14.937 \\
REGIONAL NORTE & 7.180 & 9.080 & 9.092 & 9.087 \\
REGIONAL ORIENTE & 7.148 & 9.208 & 9.246 & 9.280 \\
REGIONAL NOROESTE & 6.541 & 9.845 & 9.975 & 10.212 \\
REGIONAL VIEJO & 8.464 & 11.117 & 10.009 & 10.142 \\
CALDAS & 63.311 & 80.780 & 81.486 & 81.914 \\
\multicolumn{1}{c}{ TOTAL MES } & & JUNIO & JULIO & AGOSTO \\
& & & & 596
\end{tabular}

Fuente: Oficina de estadística INPEC, 2010

También se evaluó el potencial ambiental, social y económico de la Colonia Agrícola. Este primer insumo nos permite avanzar en una segunda fase, donde se construye el Modelo Intervención Terapéutica, basado en la Actividad Ocupacional, denominada Ganadería con bases Agroecológicas, el cual inicia con una evaluación de ingreso a las personas privadas de la libertad, para determinar su condición académica, social, judicial, personal y espiritual, para así mismo direccionar su plan de cambio o de tratamiento Penitenciario. 
Seguidamente se inicia el proceso con un momento llamado inducción a tratamiento, donde se inicia la Escuela de Capacitación en Ganadería con bases Agroecológicas, convirtiéndose en un espacio de educación integral, con un objetivo claro, la promoción de arreglos silvopastoriles en los sistemas de producción, este proceso dura tres meses. Luego viene una etapa de asignación de actividad ocupacional relacionada al tema de ganadería agroecológica, donde el interno continúa recibiendo capacitación, pero enfocada solamente a la comprensión de la agroecología como sistema productivo y social, este proceso dura tres meses. La última etapa de su proceso es la preparación para su vida en sociedad de nuevo en la libertad, en esta etapa se capacita al interno en estrategias empresariales y manejo de tecnologías, con la única intención de convertirlo en productor-empresario, para de esta manera romper el ciclo de la reincidencia en las cárceles de Colombia

\section{ANÁLISIS DEL MODELO DE INTERVENCIÓN TERAPÉUTICA CON BASE EN GANADERÍA AGROECOLÓGICA}

La experiencia en la implementación de arreglos silvopastoriles en comunidades penitenciarias ha sido un éxito, por su metodología, la cual ha incluido la educación y capacitación como el pilar más interesante del proceso; el ejercicio de la implementación de arreglos silvopastoriles son el producto práctico de la teoría entregada en los procesos de enseñanza. El modelo, su análisis y evaluación, se describe en la Gráfica 1, Fotografías 1 y 2.

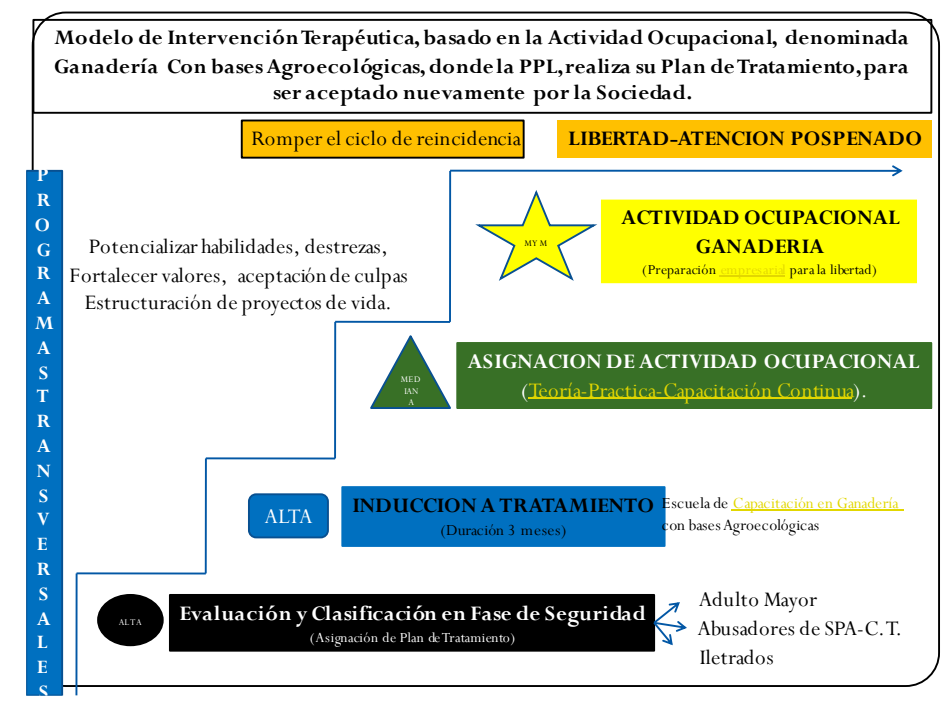

Fuente: Martínez, 2007.

Gráfica 1. Esquema del modelo de intervención terapéutica con base en ganadería agroecológica 


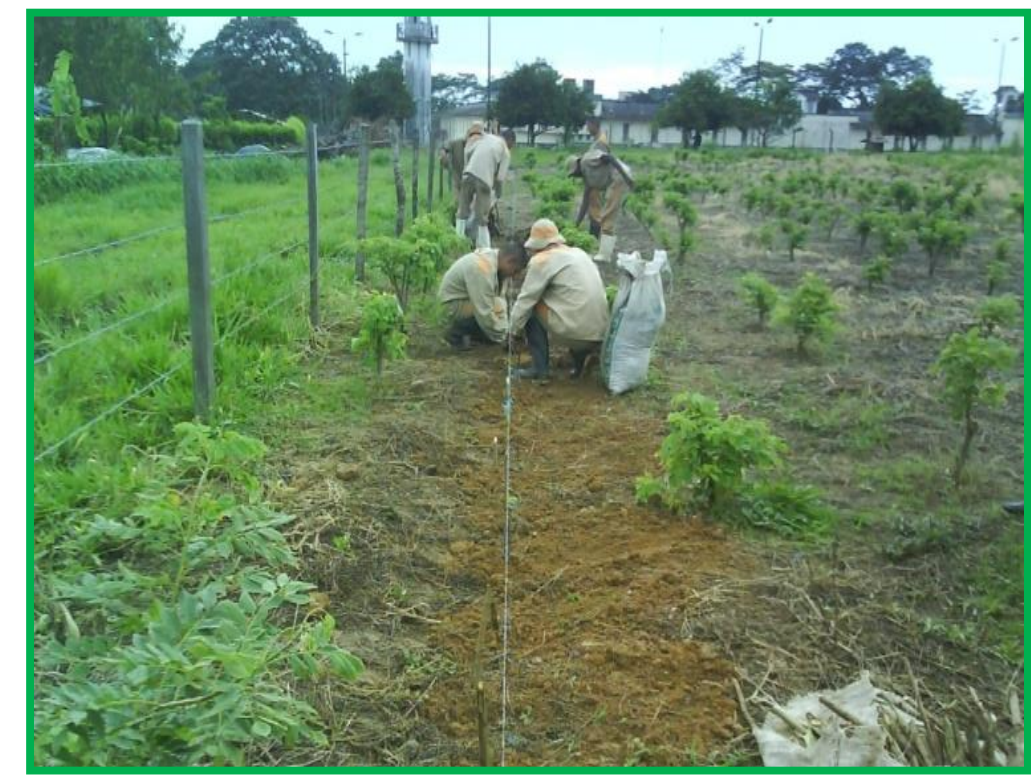

Fuente: Subdirección de Tratamiento y Desarrollo, (2009).

Fotografía 1. Internos sembrando G. sepium y Trichantera gigantea, como banco de proteína

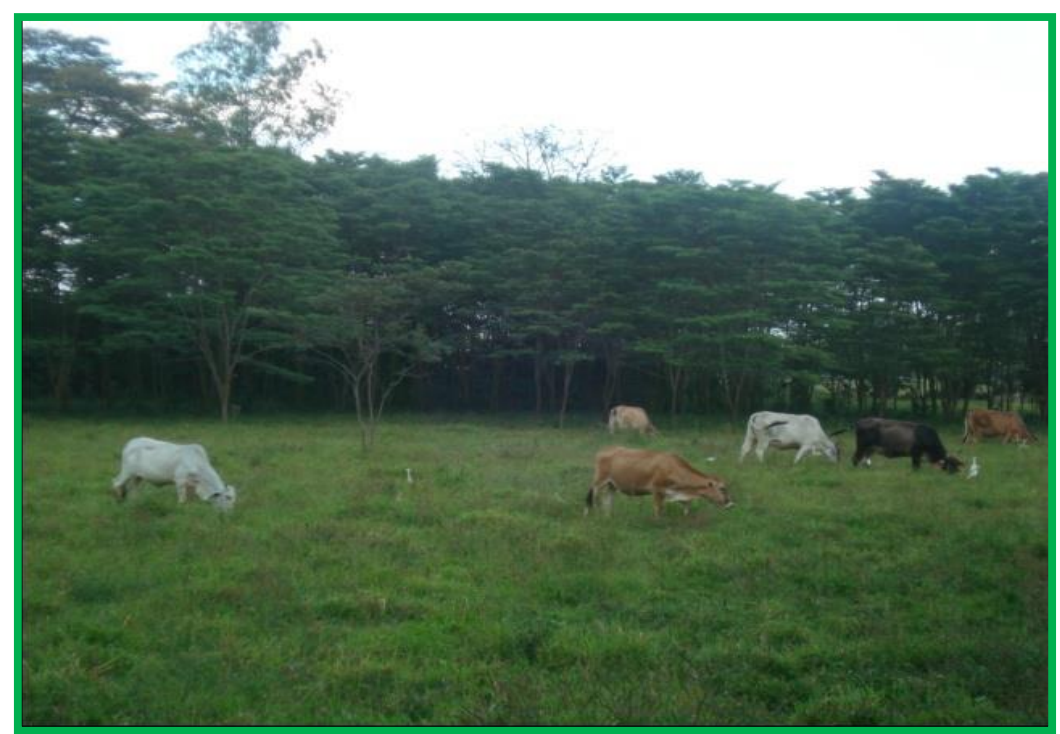

Fuente: Subdirección de Reinserción Social, (2010).

Fotografía 2. Arreglos silvopastoriles, pradera campamento cola de pato (Anadenanthera peregrina y Ceratoniasiliqua)

Otro indicador de evaluación del modelo, fue la reducción significativa del uso y abuso de sustancias psicoactivas al interior de los patios de reclusión, esta acción asociada al buen comportamiento, cambio de léxico, cambio en la presentación personal, respeto total a las autoridades.

En el tema técnico se logró garantizar la nutrición y alimentación adecuada a la población bovina de la colonia, con excelente disposición de alimento, se mejoró las tasas de producción y reproducción del hato. 


\section{LECCIONES APRENDIDAS}

La problemática social ha convertido la "CÁRCEL", en un lugar perfecto para iniciar, continuar o fortalecer los procesos de desarrollo socio-económico, mediante la capacitación de un arte u ocupación, que permita la transformación de la persona infractora y de esta manera poder romper con el ciclo vicioso de la reincidencia, que cada día es mayor y con menos intenciones reales del estado, frente a una solución social definitiva.

Hay que incluir la cárcel, en las decisiones productivas del país. La problemática de las Cárceles no es solamente del INPEC o del Estado, es de todos los colombianos.

El Modelo de Intervención Terapéutica, basado en la Actividad Ocupacional, denominada Ganadería con bases Agroecológicas, requiere un apoyo económico, para que pueda convertirse en la mejor herramienta de promoción sostenible.

Los resultados de los sistemas de producción no solamente son medibles en valor económico, sino que también existen otras intenciones como el fortalecimiento de un buen habito, que posiblemente no se le encuentra valor tangible.

\section{REFERENCIAS BIBLIOGRÁFICAS}

1. Martínez, B. La Paz en Colombia se encuentra en las Cárceles 2007. Sp

2. Pinheiro, M L.C. Pastoreo rotacional Voisín. Tecnología agroecológica para el tercer milenio. $1^{\circ}$ ed. Buenos Aires: Editorial Hemisferio Sur. 2004. 336 p.

3. Primavesi A. Manejo ecológico del suelo tropical. Memorias de un seminario. IICA. Bogotá, Febrero 26 - 28 de 2002: 51.

4. Sadeghian S, Rivera JM, Gómez ME. Impacto de la ganadería sobre las características físicas, químicas y biológicas de suelos en los andes de Colombia. En: Memorias de la conferencia electrónica sobre agroforestería para la producción animal en América Latina, realizada de abril a septiembre de CIPAV-FAO 1998:123-141.

5. Sánchez; L. Desarrollo de sistemas agroforestales en la zona sur del estado Táchira. Simposio - Taller: Experiencias en Agroforestería ejecutadas o en proceso por el INIA- Instituto Nacional de Investigaciones Agrícolas (INIA). Venezuela Táchira. 2006: 6.

6. Vega, L.E. Seminario taller sobre metodologías para la evaluación de sistemas. 1992. 